thrombolytic pathways, the induction of DAH by pristane was unaffected by the absence of plasminogen activator inhibitor1 or tissue plasminogen activator, suggesting that protection is related to the action of Serp-1 on macrophage function.

Conclusions Serp-1 blocks pristane-induced lung hemorrhage by enhancing LXR-regulated M2 macrophage polarization and Klf4-regulated IL-10 production. In view of the similarities between DAH in pristane-treated mice and SLE patients, clinical trials of Serp-1 for DAH in SLE may be warranted. Since Serp-1 treatment increases expression of the reverse cholesterol transporter ABCA1, it also may have beneficial effects on atherosclerosis in SLE patients. Supporting the feasibility of future clinical studies in SLE, Serp-1 treatment reduced myocardial damage in patients with acute coronary syndrome.

Acknowledgments This work was supported by NIH grant R01-AR44731. We are grateful to Dr. Alexandra Lucas (Arizona State University) for providing recombinant Serp-1 protein.

\section{PRISTANE INDUCED LUPUS IN HDAC6-MICE}

Christopher M Reilly. Edward Via College of Osteopathic Medicine, Blacksburg, Virginia, USA

\subsection{6/lupus-2021-lupus21 century.37}

Background We and others have reported that selective histone deacetylase 6 (HDAC6) inhibition decreases inflammation in a variety of animal models including inflammatory arthritis and lupus. In this study, we explored whether HDAC6 gene deletion could affect pristane-induced lupus.

Methods C57BL/6 (w.t.) and HDAC6 ${ }^{-/}$C57BL/6 mice were given $0.5 \mathrm{ml}$ of pristine intraperitonealy (ip) at 3 months of age. Injection of pristane induces a lupus-like syndrome whose pathogenesis implicates the secretion of type I IFN by CD11b (+) Ly6C(high) inflammatory monocytes in a TLR7-dependent fashion. Two weeks after pristane administration the mice were euthanized and assessed for various parameters of inflammation. At termination of the experiment, serum, peritoneal macrophages, and splenic tissue was collected and evaluated.

Results The pristine treated animals euthanized showed diffuse alveolar hemorrhage, both w.t. and knock-outs. At sacrifice, spleens were significantly larger in the HDAC6 $^{-/}$ mice compared to the w.t. pristine mice, however when compared to body weights there was not a significant difference. Flow cytometry did not indicate any differences in $\mathrm{T}$ cell or B cell populations. Sera IL-12, IL-6, TGF- $\beta$, IL-10, and TNF- $\alpha$ were comparable in the w.t. and the knock-out animals treated with pristine. Peritoneal recruitment of CD11b(+) Ly6C(high) inflammatory monocytes in HDAC6 ${ }^{-1}$ mice was significantly increased compared to the w.t. mice. Evaluation of the transcripts for several IFN inducible genes revealed significantly increased IRF-7 expression in the $\mathrm{HDAC}^{-/-}$mice however lower expression of IFN $\beta$ and IRF-9.

Conclusions Taken together, our results show that in early lupus induction with pristine, HDAC6 gene deletion alters monocyte activation and differentially regulates IFN inducible genes.

\section{0 - Pharmacoepidemiology}

\section{FACTORS ASSOCIATED WITH SLE FLARES AFTER HCQ TAPER, DISCONTINUATION OR MAINTENANCE IN THE SLICC INCEPTION COHORT: LOWER EDUCATION LINKED WITH HIGHER FLARE RISK}

${ }^{1}$ Celline C Almeida-Brasil, ${ }^{2} J o h n$ G Hanly, ${ }^{3}$ Murray B Urowitz, ${ }^{4}$ Ann E Clarke, ${ }^{5}$ Rosalind Ramsey-Goldman, ${ }^{6}$ Caroline Gordon, ${ }^{7}$ Michelle A Petri, ${ }^{8}$ Ellen M Ginzler, ${ }^{9}$ Daniel J Wallace, ${ }^{10}$ Sang-Cheol Bae, ${ }^{11}$ Juanita Romero-Diaz, ${ }^{12}$ Mary Anne Dooley, ${ }^{13}$ Christine A Peschken, ${ }^{14}$ David A Isenberg, ${ }^{14}$ Anisur Rahman, ${ }^{15}$ Susan Manzi, ${ }^{16}$ Soren Jacobsen, ${ }^{17}$ Sam Lim, ${ }^{18}$ Ronald van Vollenhoven, ${ }^{19}$ Ola Nived, ${ }^{19}$ Andreas Jonsen, ${ }^{20}$ Diane L Kamen, ${ }^{21}$ Cynthia Aranow, ${ }^{22}$ Guillermo Ruiz-Irastorza, ${ }^{3}$ Jorge Sanchez-Guerrero, ${ }^{3}$ Dafna D Gladman, ${ }^{23}$ Paul R Fortin, ${ }^{24}$ Graciela S Alarcón, ${ }^{25}$ Joan T Merrill, ${ }^{26}$ Kenneth C Kalunian, ${ }^{27}$ Manuel Ramos-Casals, ${ }^{28}$ Kristjan Steinsson, ${ }^{29}$ Asad Zoma, ${ }^{30}$ Anca Askanase, ${ }^{31}$ Munther A Khamashta, ${ }^{32}$ Ian Bruce, ${ }^{33}$ Murat Inanc, ${ }^{1}$ Sasha Bernatsky*. ${ }^{1}$ Research Institute of the McGill University Health Centre, Canada; ${ }^{2}$ Queen Elizabeth II Health Sciences Centre, Canada; ${ }^{3}$ University of Toronto, Canada; ${ }^{4}$ University of Calgary, Canada; ${ }^{5}$ Northwestern University, USA; ${ }^{6}$ Institute of Inflammation and Ageing of the University of Birmingham, UK; ${ }^{7}$ Johns Hopkins University School of Medicine, USA; ${ }^{8}$ SUNY Downstate Medical Center, USA; ${ }^{9}$ Cedars-Sinai Medical Centre, USA; ${ }^{10}$ Hanyang University Hospital for Rheumatic Diseases, South Korea; ${ }^{11}$ Instituto Nacional de Ciencias Médicas y Nutrición, Mexico; ${ }^{12}$ UNC Kidney Centre, USA; ${ }^{13}$ University of Manitoba, Canada; ${ }^{14}$ University College London, UK; ${ }^{15}$ Allegheny Health Network, USA; ${ }^{16}$ Rigshospitalet, Denmark; ${ }^{17}$ Emory University School of Medicine, USA; ${ }^{18}$ University of Amsterdam, Netherlands; ${ }^{19}$ Lund University, Sweden; ${ }^{20}$ Medical University of South Carolina, USA; ${ }^{21}$ Feinstein Institute for Medical Research, USA; ${ }^{22}$ Hospital Universitario Cruces, Spain; ${ }^{23}$ Université Laval, Canada; ${ }^{24}$ University of Alabama at Birmingham, USA; ${ }^{25}$ Oklahoma Medical Research Foundation, USA; ${ }^{26}$ UC San Diego School of Medicine, USA; ${ }^{27}$ Universitat de Barcelona, Spain; ${ }^{28}$ Landspitali University Hospital, Iceland; ${ }^{29}$ Hairmyres Hospital, UK; ${ }^{30}$ Columbia University Medical Centre, USA; ${ }^{31}$ St Thomas' Hospital, UK; ${ }^{32}$ University of Manchester, UK; ${ }^{33}$ Istanbul University, Turkey

\subsection{6/lupus-2021-lupus21century.38}

Background Hydroxychloroquine (HCQ) is a cornerstone treatment of systemic lupus erythematosus (SLE). We compared time to flare in SLE patients discontinuing/reducing HCQ versus those maintaining their dose, and identified factors associated with time to flare.

Methods We analyzed prospective data from the Systemic Lupus International Collaborating Clinics (SLICC) cohort, which includes SLE patients from 33 sites in Europe, Asia, and North America, enrolled within 15 months of diagnosis and followed annually (1999-2019). We identified patients with HCQ reduction/discontinuation, regardless of disease activity. We evaluated person-time that patients contributed on their initial dose ('maintenance'), comparing this to persontime contributed after a first dose reduction, and person-time after a first HCQ discontinuation. We estimated time to first flare, defined as either subsequent need for therapy augmentation (steroids or other immunomodulators), increase of $\geq 4$ points in the SLE Disease Activity Index-2000 (SLEDAI-2K) or hospitalization for SLE. We estimated crude flare rates for each sub-cohort and hazard ratios and 95\% confidence intervals (CIs) for various demographic and clinical factors potentially associated with flare risk in the reduction and discontinuation sub-cohorts, as well as comparator maintenance sub-cohorts (matched for time on HCQ to the reduction and discontinuation sub-cohorts).

Results We studied 1460 SLE patients (90\% women, 52\% Caucasian) on HCQ. Of these, 592 subsequently reduced HCQ at any point, while 407 discontinued HCQ at any point. The crude flare rate for the HCQ reduction sub-cohort was 42.3 per 100 person-years (95\% CI 38.6, 46.4), versus 35.6 (95\% CI $32.4,39.1)$ in the matched maintenance subcohort. In the discontinuation sub-cohort, the crude flare rate 